\title{
PDLSCs Regulate Angiogenesis of Periodontal Ligaments via VEGF Transferred by Exosomes in Periodontitis
}

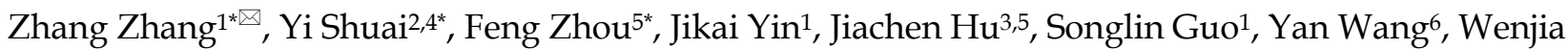 \\ Liu $^{3,5}$ \\ 1. Department of General Surgery, Tang Du Hospital, Fourth Military Medical University, Xi'an, Shaanxi 710032, People's Republic of China; \\ 2. Department of Stomatology, Jinling Hospital, Medical School of Nanjing University, Nanjing, Jiangsu 210002, People's Republic of China; \\ 3. State Key Laboratory of Military Stomatology \& National Clinical Research Center for Oral Diseases \& Shaanxi International Joint Research Center for Oral \\ Diseases, Center for Tissue Engineering, School of Stomatology, Fourth Military Medical University, Xi'an, Shaanxi 710032, People's Republic of China; \\ 4. Department of Stomatology, General Hospital of Eastern Theater Command, PLA, Nanjing, Jiangsu 210002, People's Republic of China; \\ 5. Xi'an Institute of Tissue Engineering and Regenerative Medicine, Xi'an, Shaanxi 710032, People's Republic of China; \\ 6. Department of Clinical Laboratory, The First Affiliated Hospital of Xi'an Medical University, Xi' an, Shaanxi 710032, People's Republic of China. \\ *These authors contributed equally to the study. \\ $\triangle$ Corresponding authors: Wenjia Liu, MD. PhD. Tel: +86-29-84776147, Fax: +86-29-83218039, E-mail: wenjia@xiterm.com; wenjialiu23@163.com; Zhang Zhang, \\ MD. PhD. Tel: +86-29-84778265, Fax: +86-29-83218039, E-mail: pwzhangz@fmmu.edu.cn; zhangz0613@163.com.
}

(C) The author(s). This is an open access article distributed under the terms of the Creative Commons Attribution License (https://creativecommons.org/licenses/by/4.0/). See http://ivyspring.com/terms for full terms and conditions.

Received: 2019.10.05; Accepted: 2020.01.08; Published: 2020.02.10

\begin{abstract}
Abnormal angiogenesis is one of the significant features in periodontitis leading to progressive inflammation, but angiogenic changes of periodontal ligaments under inflammatory condition were rarely reported. Periodontal ligament stem cells (PDLSCs) were a kind of dental stem cells associated with vascularization. Here we investigated the alteration of angiogenesis of periodontal ligament in periodontitis, and revealed an exosome-mediated pathway to support the effect of PDLSCs on angiogenic improvement. Vascular specific marker CD31 and VEGFA were found to be highly expressed in periodontal ligaments of periodontitis. The VEGFA expression was up-regulated in inflamed PDLSCs compared to control, meanwhile the tube formation of HUVECs was improved when co-cultured with inflamed PDLSCs. Exosomes secretion of PDSLCs was augmented by inflammation, and promoted angiogenesis of HUVECs, whereas blocking secretion of exosomes led to degenerated angiogenesis of HUVECs. Exosome-trasferred VEGFA was proven to be the crucial communicator between PDLSCs and HUVECs. Inflammation inhibited miR-17-5p expression of PDLSCs and relieved its target VEGFA. However, overexpression of miR-17-5p blocked the pro-angiogenic ability of inflamed PDLSCs. In conclusion, the findings indicated that vascularization of periodontal ligaments was enhanced, and inflammatory micro-environment of periodontitis facilitated pro-angiogenesis of PDLSCs through regulating exosome-mediated transfer of VEGFA, which was targeted by miR-17-5p.
\end{abstract}

Key words: periodontitis, angiogenesis, PDLSCs, exosomes, miR-17-5p, VEGF

\section{Introduction}

Periodontitis is identified as a chronic bacterial infectious disease, which invades the toothsupporting tissues, such as gingiva, periodontal ligaments and alveolar bone [1]. The growth and remodeling of the skeletal system are generally coupled with angiogenesis, including alveolar bone [2]. Anomalous vascularization is one of the most common characteristics in periodontitis leading to progressive inflammation, which results from disrupted angiogenesis in the periodontal tissues [3]. Recently, large numbers of studies on periodontitis have been concentrating on angiogenesis in the inflammatory tissues [4]. Increased blood vessels were observed in gingiva of the periodontitis as a result of enhanced inflammatory infiltration [5, 6]. Periodontal ligament locates in the gap between tooth root and 
alveolar bone to fasten the tooth, which is often invaded and destructed by inflammation when chronic periodontitis was extending [7]. Therefore it might be very important to determine the alteration of periodontal ligament for uncovering pathogenesis of periodontitis. However, changes of vascularization in periodontal ligament were rarely reported in the condition of periodontitis, and detailed mechanisms remain to be revealed.

Mesenchymal stem cells (MSCs) have been widely reported to be associated with angiogenesis, including dental stem cells $[4,8,9]$. Periodontal ligament stem cells (PDLSCs) are a class of odontogenic MSCs residing in periodontal ligament with functions of self-renewal, multi-directional differentiation potential and cellular regulation [10]. Recent study reported that PDLSCs had an ability of angiogenic promotion $[4,9]$, whereas the underlying mechanism of PDLSCs on angiogenesis under inflammatory micro-environment needed to be uncovered.

Paracrine is an important mode of intercellular communication, which is achieved by secreting soluble factors, protein complex and packaged mediators [11, 12]. Exosomes, a type of packaged mediators probably containing functional proteins, functionally transfers to other cells through such an emerging novel paracrine pathway [12, 13]. Numerous evidences showed that MSCs, as well as dental stem cells, could regulate function of endothelial cells via exosome-mediated signaling [14, 15]. Various biological factors were involved in angiogenic regulation [16]. However, the vascular endothelial growth factor (VEGF) was commonly regarded as the most potent agent participating in modulation of vascular endothelium [17]. Furthermore, VEGF was reported to be a major stimulant in the process of coupling between osteogenesis and angiogenesis $[18,19]$.

Numerous micro-RNAs (miRNAs) were investigated to play significant roles in dental tissues [20]. MiR-17, an inhibitor of VEGF, is a crucial microRNA associated with bone remodeling and angiogenesis [21-24]. Our previous study demonstrated that miR-17 regulated periodontal tissue regeneration via PDLSCs-mediated pathway in chronic periodontitis $[21,22,24]$. Therefore, current work was assumed to explore whether PDLSCs can regulate angiogenesis via exosome-mediated VEGF communication under inflammatory environment of periodontitis.

In current study, enhanced vascularization was found in periodontal ligament, as well as gingiva, derived from chronic periodontitis. PDLSCs cultured in inflammatory condition expressed more VEGFA and promoted tube formation of human umbilical vein endothelial cells (HUVECs). Additionally, blocking of exosomes secretion suppressed the tube formation, and inflammation inhibited expression of miR-17-5p while improved the VEGFA expression in exosomes. All the observations suggested that PDLSCs, under inflammatory condition of periodontitis, regulated angiogenesis through exosomemediated transportation of VEGFA targeted by miR-17-5p, which uncovered a novel mechanism of aberrant vascularization in periodontal ligaments of periodontitis and proposed a therapeutic target for periodontitis.

\section{Materials and Methods}

\section{Animal model of periodontitis}

Twenty adult female Sprague-Dawley rats $(200.4 \pm 25.3 \mathrm{~g})$ were purchased from the Laboratory Animal Research Centre of the Fourth Military Medical University. Periodontitis was induced by injection of Escherichia Coli LPS $(10 \mu \mathrm{L}, 1 \mathrm{mg} / \mathrm{mL})$ at the mediolateral of the left mandibular first molar, while the control group received $10 \mu \mathrm{L}$ of saline. This administration was repeated every other day until 30 days. All the procedures were approved by Animal Care Committee of the Fourth Military Medical University, following the NIH Guide for the Care and Use of Laboratory Animals.

\section{Micro-CT analysis}

The micro-CT system (L-sp, GE, USA) was applied to scan the mandible samples with a source voltage of $80 \mathrm{kV}$, current of $500 \mathrm{~mA}$ and isotropic resolution of $14.97 \mathrm{~mm}$. Three-dimensional images of the periodontal defects were reconstructed by the supporting software of micro-CT.

\section{Immunofluorescent analysis}

Frozen sections of periodontal tissues were prepared for immunofluorescent staining. The gingiva and periodontal ligament were tested by immunofluorescence against CD31 (ab119339) and VEGF (ab46154) (Abcam, USA) 30 days after induction of periodontitis. Sections were stained using primary antibodies at $4^{\circ} \mathrm{C}$ overnight. The nuclei were then stained using DAPI at $37^{\circ} \mathrm{C}$ for $30 \mathrm{~min}$ after incubation with secondary antibodies of Alexa Fluor 488 and Alexa Fluor 594.

\section{Patients and tissue samples}

Impacted premolars of patients were collected at the School of Stomatology, Fourth Military Medical University, whose teeth were extracted because of orthodontic purposes or periodontitis. All the procedures of human samples were approved by the Institutional Review Board for Human Subjects Research of Fourth Military Medical University 
(IRB-REV-2016021). All the patients signed informed consent to this study.

\section{Cell isolation, culture and differentiation assay}

Periodontal ligaments derived from normal and periodontitis donors were separated from the middle of root surfaces, then were cut into small pieces (about $1 \mathrm{~mm}^{3}$ ) and digested using collagenase type I (3 $\mathrm{mg} / \mathrm{mL})$, dispase $(4 \mathrm{mg} / \mathrm{mL})$ (Sigma Aldrich, USA) for $15 \mathrm{~min}$. Single-cell suspensions $\left(2 \times 10^{3}\right.$ cells $)$ were seeded and cultured in a-MEM (Gibco, USA) with 10\% FBS (Gibco), streptomycin (100 IU/ml; Gibco) and penicillin $(100 \mu \mathrm{g} / \mathrm{ml}$; Gibco $)$ with an environment of $37^{\circ} \mathrm{C}$ and humidified $5 \% \quad \mathrm{CO}_{2}$. PDLSCs were cultured in basic medium until reaching $80 \%$ confluence, then were induced by osteogenic condition medium for 28 days or adipogenic condition medium for 14 days. Alizarin Red and Oil O Red were used for staining of mineralized nodules or lipid droplets. The $2^{\text {nd }}-5^{\text {th }}$ passages of PDLSCs were used for each experiment.

\section{Flow cytometry}

The single-cell suspension of $5 \times 10^{5}$ PDLSCs (P5) was incubated with antibodies for human Stro-1 (PE), CD146 (PE), CD105 (PE), CD29 (FITC), CD34 (PE) and CD45 (APC) (BD Bioscience, USA) at $4^{\circ} \mathrm{C}$ without light. The samples were detected by flow-cytometry using a Beckman Coulter Epics XL cytometer (Beckman Coulter, USA) to identify stem cell surface markers.

\section{Exosomes isolation}

PDLSCs were cultured with vesicle-free medium and serum. The residual cells were removed from harvested conditioned medium by centrifuging for 10 min at $500 \mathrm{~g}$. Next, the apoptotic bodies and debris were removed by centrifugation of the supernatant for $30 \mathrm{~min}$ at $16,000 \mathrm{~g}$. A following ultracentrifugation for $70 \mathrm{~min}$ at 150,000 $\mathrm{g}$ (Beckman Optima XPN-100, USA) was conducted to collect exosomes. The exosomes were stored at $80^{\circ} \mathrm{C}$ in $\mathrm{PBS}$ for further research.

\section{Morphological examination of exosomes}

The isolated exosomes were suspended and dyed using Phosphotungstic Acid (PTA). The morphology of exsomes was observed and captured by transmission electron microscopy (Tecnai G2, USA).

\section{Tube formation assay}

Matrigel (BD Biosciences, USA) and a 24-well transwell plate (Corning, NY) were used for tube formation assay. $2 \times 10^{4}$ HUVECs per well were seeded in matrigel-coated 24-well plate, and indirectly co-cultured with $1 \times 10^{4}$ PDLSCs or $50 \mu \mathrm{g} / \mathrm{mL}$ exosomes for $5 \mathrm{~h}$ at $37^{\circ} \mathrm{C}$. After incubation, tube formation was determined under optical microscope (Leica, Germany). The network structures of tube formation were measured using Image J software.

\section{Transfection of miRNA mimics and inhibitor}

Cells were used for transfection when reached to $65 \%$ confluence. PDLSCs were transfected with miR-17-5p mimics at $20 \mathrm{nM}$ and inhibitor at $60 \mathrm{nM}$ (RiboBio, China) using Ribo FECT CP Transfection Kit (RiboBio, China) according to the instructions. The transfection medium was replaced after $8 \mathrm{~h}$, and the cells were collected for protein analysis after $48 \mathrm{~h}$.

\section{Luciferase Reporter Assay}

Luciferase reporter assay was performed as reported previously [22]. In brief, VEGFA oligonucleotide sequences were amplified using the primers (sense: 5'-ATCTTCAAGCCGTCCTGTG-3', anti-sense: 5'-GAATGGGTTTGTCGTGTTT-3') with Speland HindIII sites at their ends to insert pMIR-Report luciferase plasmid (Ambion, USA). The pMIR-control, pMIR-VEGFA plasmids were used as reporter constructs, and a Renilla luciferase reporter without miRNA binding sites was used as the loading control. All plasmids were co-transfected into PDLSCs with miR-17-5p mimics, inhibitor, or negative control using Lipofectamine 2000 (Invitrogen, USA). After $48 \mathrm{~h}$, luciferase activities were determined using a dual-luciferase reporter assay kit (Promega, USA) following the protocols.

\section{Western blot analysis}

Protein concentration measurement was conducted using a BCA kit (Beyotime Biotechnology, China). Equal protein amount from each sample was taken for western blot analysis. The proteins were transferred to PVDF membrane after separation. 5\% non-fat milk powder solution was used to block non-specific bands. Antibody of VEGF (ab46154) and TSG101 (ab125011) (Abcam, USA), GAPDH (CW0100) and $\beta$-Tubulin (CW0098) (CWBio, China), CD63 (sc-5275) (Santa Cruz Biotechnology, USA) were used for western blot. The protein bands were developed using Amersham Imager 600 (GE Healthcare Life Sciences, USA).

\section{Statistical analysis}

All the data were displayed using mean \pm SEM and were analyzed by Student's $t$ test or one-way ANOVA for comparison of two or multiple groups with SPSS software v11.0 (SPSS, USA). P $<0.05$ was considered as statistically significant. 


\section{Results}

The vascularization of gingiva and periodontal ligaments were both up-regulated in periodontitis

Micro-CT was applied to estimate the periodontitis model. Periodontal defects were observed in periodontitis group (Fig. 1A), meanwhile the crestal bone loss of periodontitis group was increased compared to control group (Fig. 1B), suggesting that periodontitis model was successfully established. Furthermore, to investigate the angiogenesis of gingiva and periodontal ligaments, tissues were stained by vascular marker CD31 and VEGFA. Compare with control group, the positive areas of CD31 and VEGFA were larger in gingiva of periodontitis group (Fig. 1C), as well as the overlapped area of the two markers. In addition, similar results were observed in periodontal ligaments (Fig. 1D), which indicated that periodontal ligaments shared similar vascular alteration that was up-regulated in periodontitis.

\section{The pro-angiogenic capacity of PDLSCs was improved under inflammatory micro-environment}

Surface markers, osteogenesis and adipogenesis of PDLSCs were identified (Fig. S1). To determine the effect of inflammatory micro-environment on pro-angiogenic capacity of PDLSCs, the expression of
A

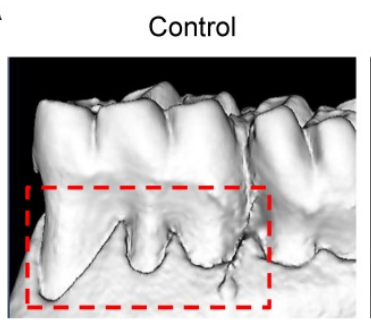

C

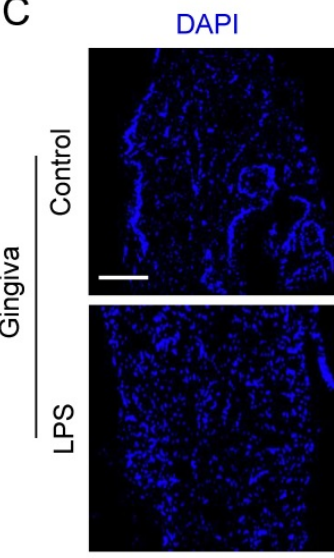

D

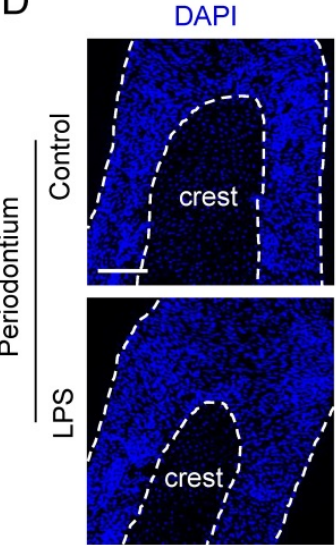

LPS

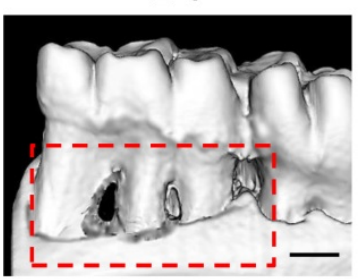

CD31
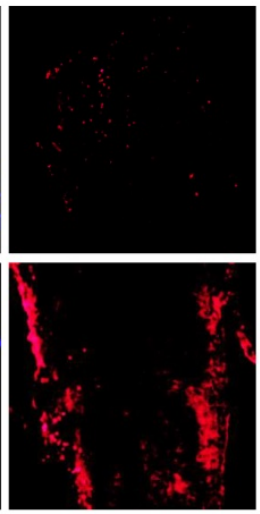

CD31
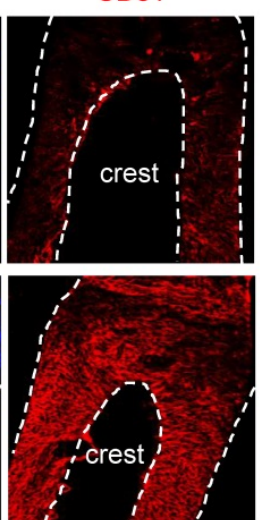

$\mathrm{B}$

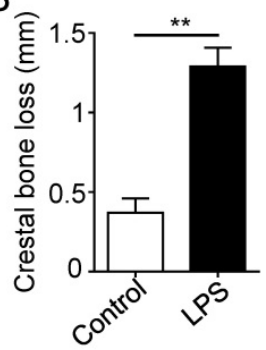

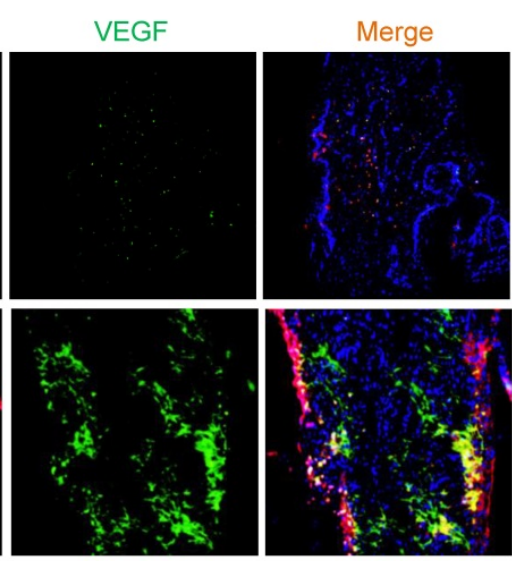
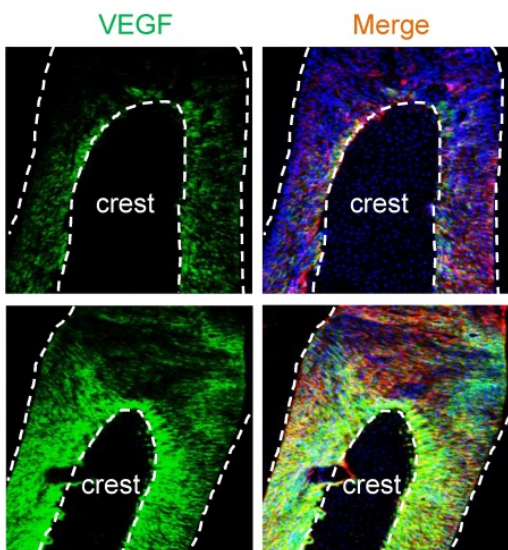

Figure 1. The angiogenesis of gingiva and periodontal ligaments in periodontitis. The alveolar bone (A) and crestal bone loss (B) were analyzed using micro- $\mathrm{CT}$ and quantitative analysis of the crestal bone loss $(n=6)$. Scale bar $=500 \mu \mathrm{m}$. Vascular markers CD31 and VEGF of gingiva (C) and periodontal ligaments $(D)$ were stained using immunofluorescent analysis. Scale bar $=200 \mu \mathrm{m}$. $* * p<0.01$. Unpaired two-tailed Student's t-test. 
VEGFA was examined using western blot. The VEGFA was up-regulated in PDLSCs derived from periodontitis and PDLSCs treated with inflammatory factors compared to control group (Fig. 2A). Moreover, a transwell co-culture system was employed to evaluate the effect of PDLSCs on HUVECs (Fig. 2B). The tube formation ability, including total tube length, total branching points, total loops, covered area and total nets, was enhanced in PDLSCs derived from periodontitis and PDLSCs treated with inflammatory factors compared to control group (Fig. $2 \mathrm{C}$ and D). Additionally, the VEGFA expression (Fig. 2A) and tube formation were much more in the group treated with inflammatory factors for 14 days than that for 7 days (Fig. 2C and D). All these results suggested that inflammation promoted the pro-angiogenesis of PDLSCs.

\section{PDLSCs promoted angiogenesis through exosome-mediated pathway under inflammatory condition}

The 14 days-treatment was used for further study because of its better effect on pro-angiogenesis

compared to the 7 days-treatment. To explore whether inflammation influenced the exosomes secretion, PDLSCs were treated with inflammatory factors. Scanning electron microscope (SEM) showed that the quantity of exosomes was increased in inflammation group compared to control group (Fig. 3A). In addition, expressions of exosomes specific markers (TSG101, CD63) were higher in inflammation group than that in control group (Fig. 3B). The aforementioned data suggested that inflammation promoted exosomes secretion of PDLSCs. Furthermore, HUVECs treated with exosomes derived from inflamed PDLSCs exhibited better tube formation than control group (Fig. 3C-E). However, knockingdown exosomes of PDLSCs using GW4869 resulted in declined tube formation of HUVECs (Fig. 4A-C) and angiogenic markers (CD31, VEGFA) expression (Fig. $4 \mathrm{D})$. Taken together, all these results indicated that PDLSCs promoted angiogenesis of HUVECs via exosome-mediated pathway under inflammatory micro-environment.
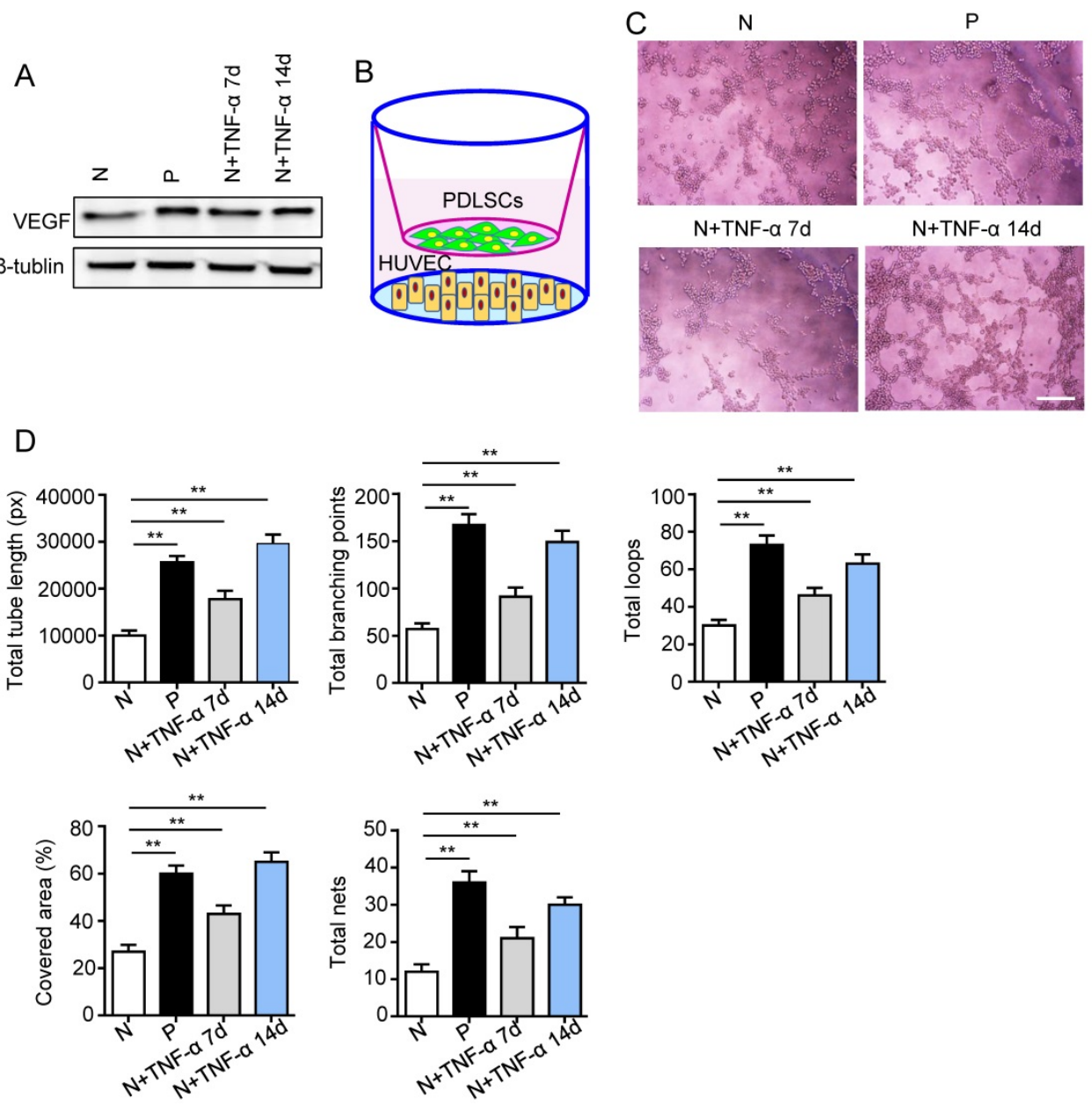

Figure 2. The pro-angiogenic capacity of PDLSCs under inflammatory micro-environment. (A) VEGF expression of PDLSCs was detected using western blot. (B) A transwell co-culture system was applied. Tube formation of HUVECs (C) was observed under microscope after co-culturing with PDLSCs, and total tube length, total branching points, total loops, covered area and total nets (D) were analyzed using Image J. Scale bar $=200 \mu \mathrm{m}$. **p<0.01. One-way analysis of variance (ANOVA). 

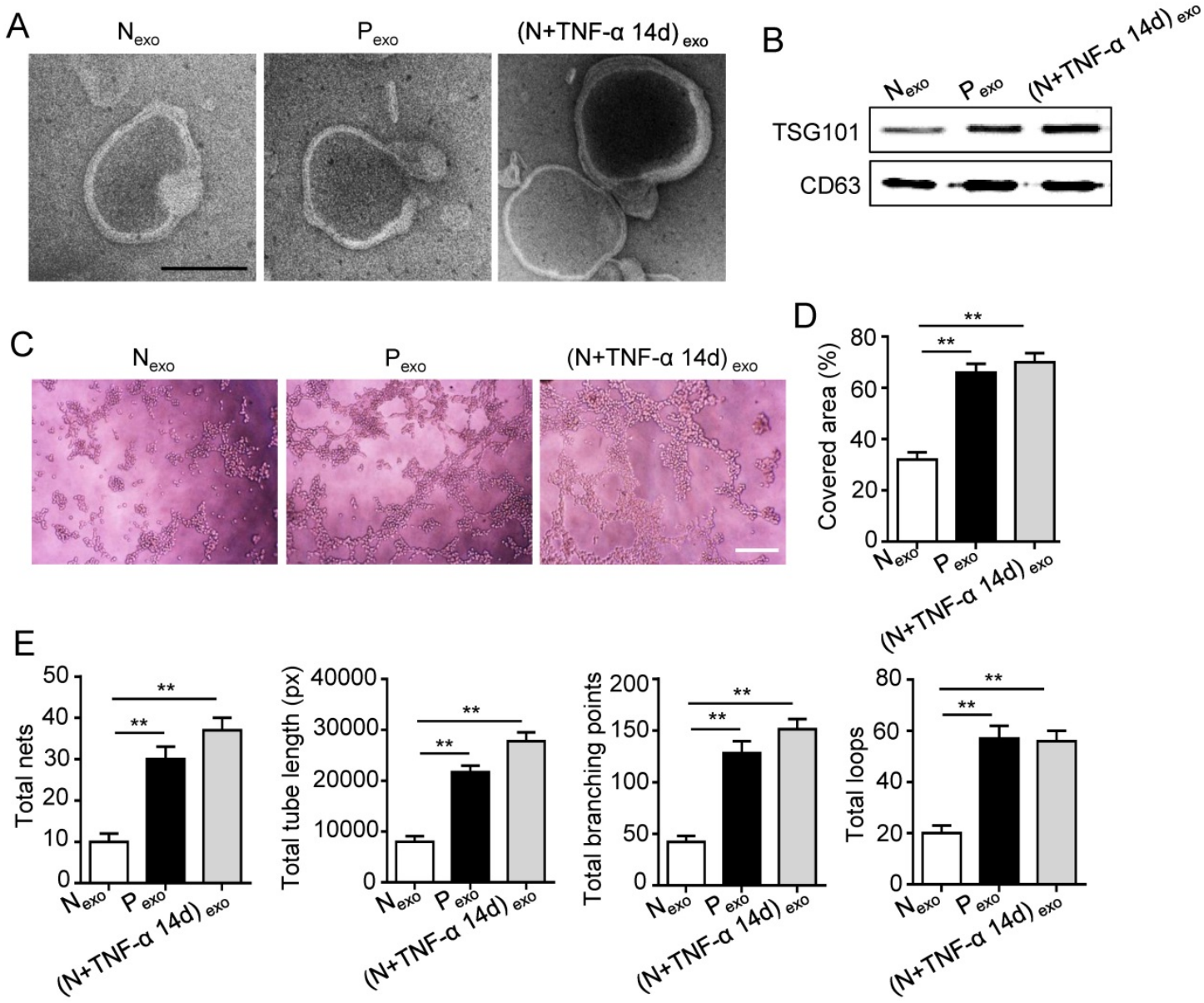

Figure 3. Effect of PDLSCs on angiogenesis through exosome-mediated pathway under inflammatory condition. (A) Exosomes secretion was detected using SEM. Scale bar $=100 \mathrm{~nm}$. (B) Exosomes specific marker TSG 101 and CD63 were examined by western blot. Tube formation of HUVECs (C) was observed under microscope after treatment with exosomes derived from PDLSCs, and total tube length, total branching points, total loops, covered area and total nets (D-E) were analyzed using Image J. Scale bar $=200 \mu \mathrm{m} . * * p<0.01$. One-way analysis of variance (ANOVA).

\section{Inflammation affected pro-angiogenesis of PDLSCs via regulating exosome-mediated transfer of VEGFA targeted by miR-17-5p}

In order to clarify whether exosomes transported VEGFA to HUVECs, proteins were analyzed by western blot. The VEGFA expression was much higher in exosomes derived from inflamed PDLSCs than control group (Fig. 5A). In addition, HUVECs also showed higher VEGFA expression when treated with exosomes derived from inflamed PDLSCs compared to normal PDLSCs (Fig. 5B), suggesting that PDLSCs transported VEGFA to HUVECs via exosomes secretion under inflammation.

Previous studies reported that miR-17 was related to angiogenesis and VEGF was a target of miR-17. To further identify the alteration of miR-17 and its effect on VEGF expression under inflammatory micro-environment, the gain and loss experiment was conducted through transfecting mimics and inhibitor of miR-17-5p into PDLSCs respectively (transfection efficiency see Fig. S2). The data showed that miR-17-5p was inhibited under inflammatory condition (Fig. 5C), but its alteration did not affect the secretion of exosomes (Fig. S3). Additionally, silence of miR-17-5p did not affect the expression of VEGFA mRNA (Fig. 5D) but significantly improved VEGFA protein expression (Fig. 5E), suggesting that miR-17-5p regulated VEGFA at post-transcriptional level. Luciferase reporter test showed that overexpression of miR-17-5 $p$ largely reduced the luciferase activity of VEGFA 3'-UTR, whereas knocking-down of miR-17 increased the reporter luciferase activity (Fig. 5F), confirming that VEGFA was a target of miR-17-5p. Furthermore, compared to control group, the VEGFA expression significantly decreased in exosomes derived from PDLSCs transfected by miR-17-5p mimics (Fig. 6A), meanwhile the tube formation of HUVECs was correspondingly declined (Fig. 6B-D). Taken together, all these data indicated that inflammation relieved the VEGFA suppression by inhibiting miR-17-5p of PDLSCs, and improved VEGFA transferring via exosomes secretion to facilitate angiogenesis of HUVECs (Fig. 6E). 

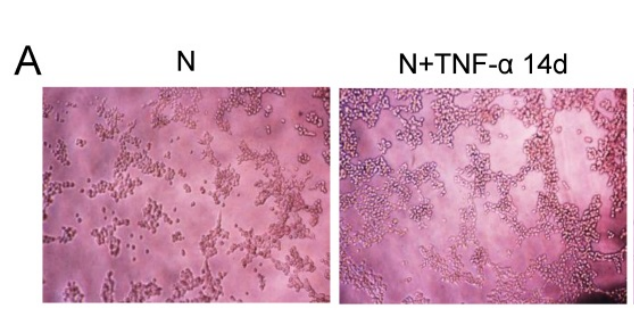

\section{$N+T N F-a 14 d$}

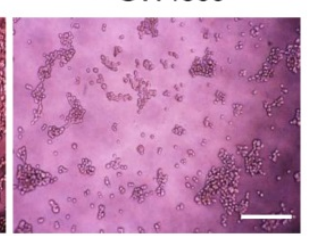

C
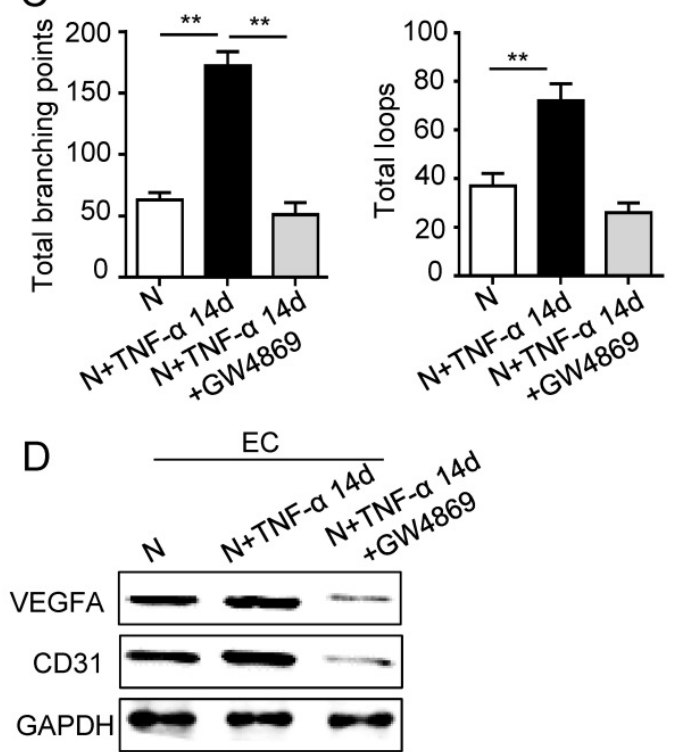

+ GW4869
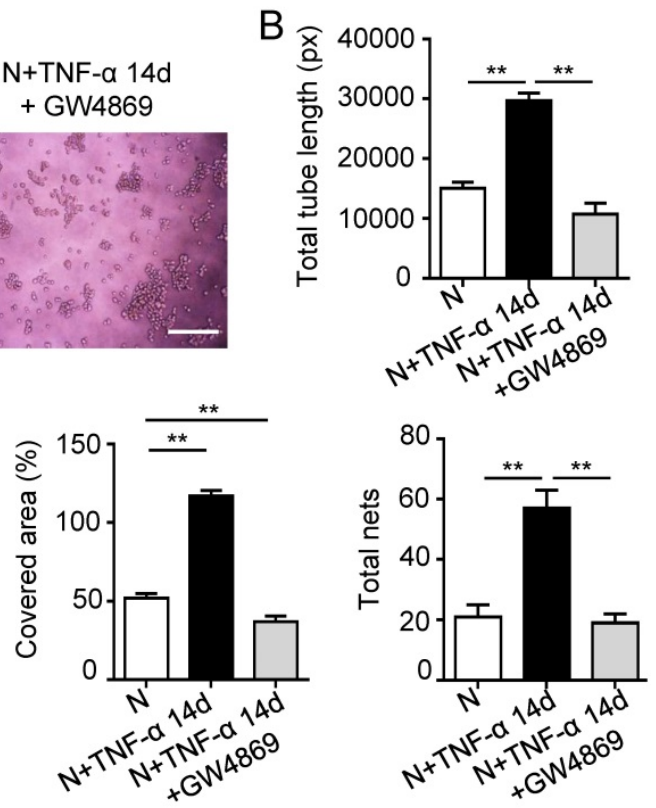

Figure 4. Effect of PDLSCs on angiogenesis when exosome-mediated pathway was blocked under inflammatory condition. Exosomes were blocked by GW4869. Tube formation of HUVECs (A) was observed under microscope, then total tube length, total branching points, total loops, covered area and total nets (B-C) were analyzed using Image J, and VEGF and CD31 of HUVECs (D) were detected by western blot after co-culturing with PDLSCs. Scale bar $=200 \mu \mathrm{m}$. $* * p<0.01$. One-way analysis of variance (ANOVA).
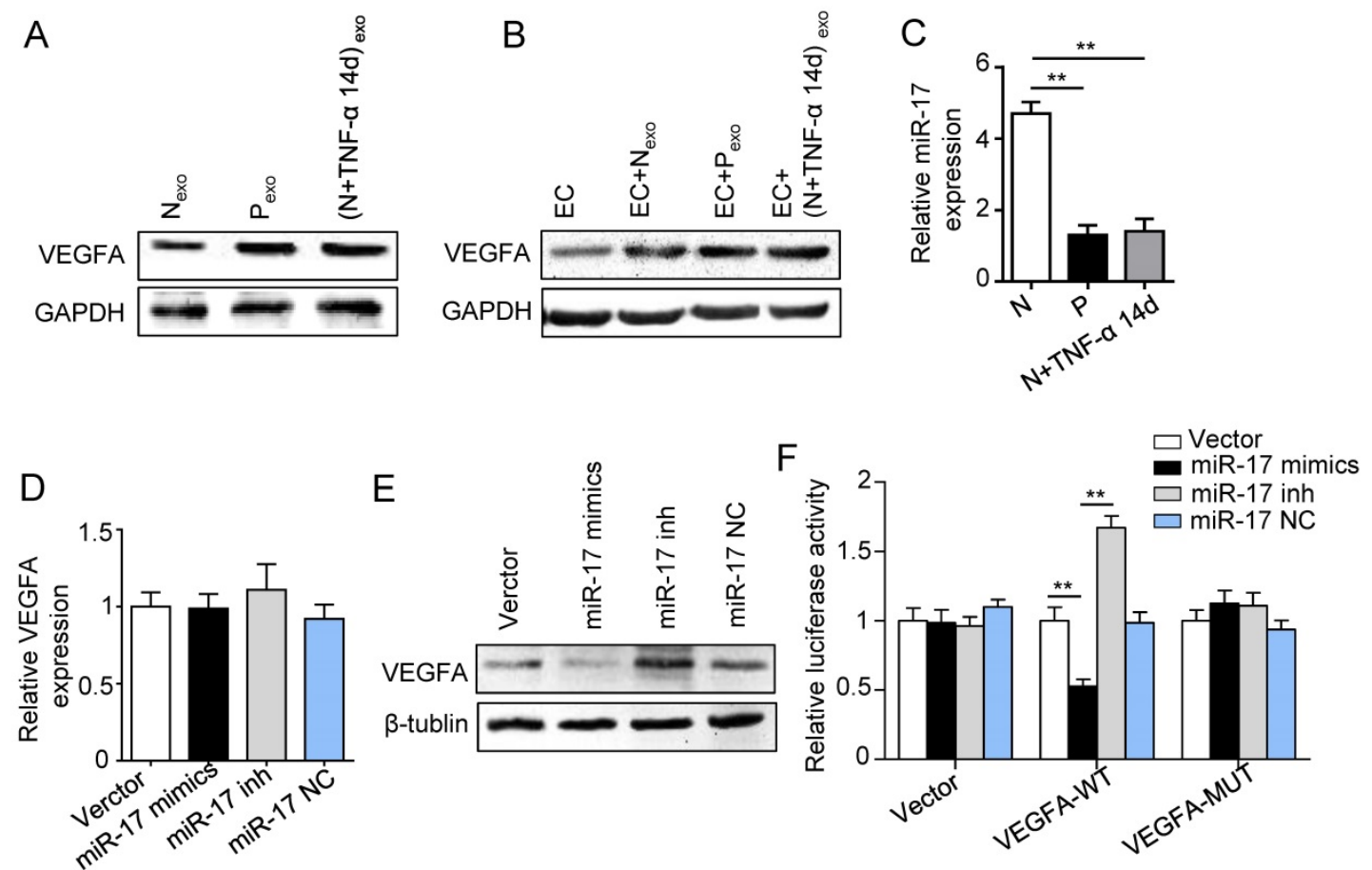

Figure 5. Effect of inflammation on VEGFA transportation from PDLSCs to HUVECs, and verification of miR-17-5p targeting VEGFA. (A) VEGFA expression in exosomes derived from was detected using western blot. (B) VEGFA expression in HUVECs was detected using western blot after treatment with exosomes derived from PDLSCs. (C) MiR-17-5p expression was detected using real-time PCR. VEGFA mRNA (D) and protein (E) were examined by real-time PCR and western blot respectively. (F) Luciferase activity of VEGF 3'-UTR was detected by luciferase report assay. $* * p<0.01$. One-way analysis of variance (ANOVA). 

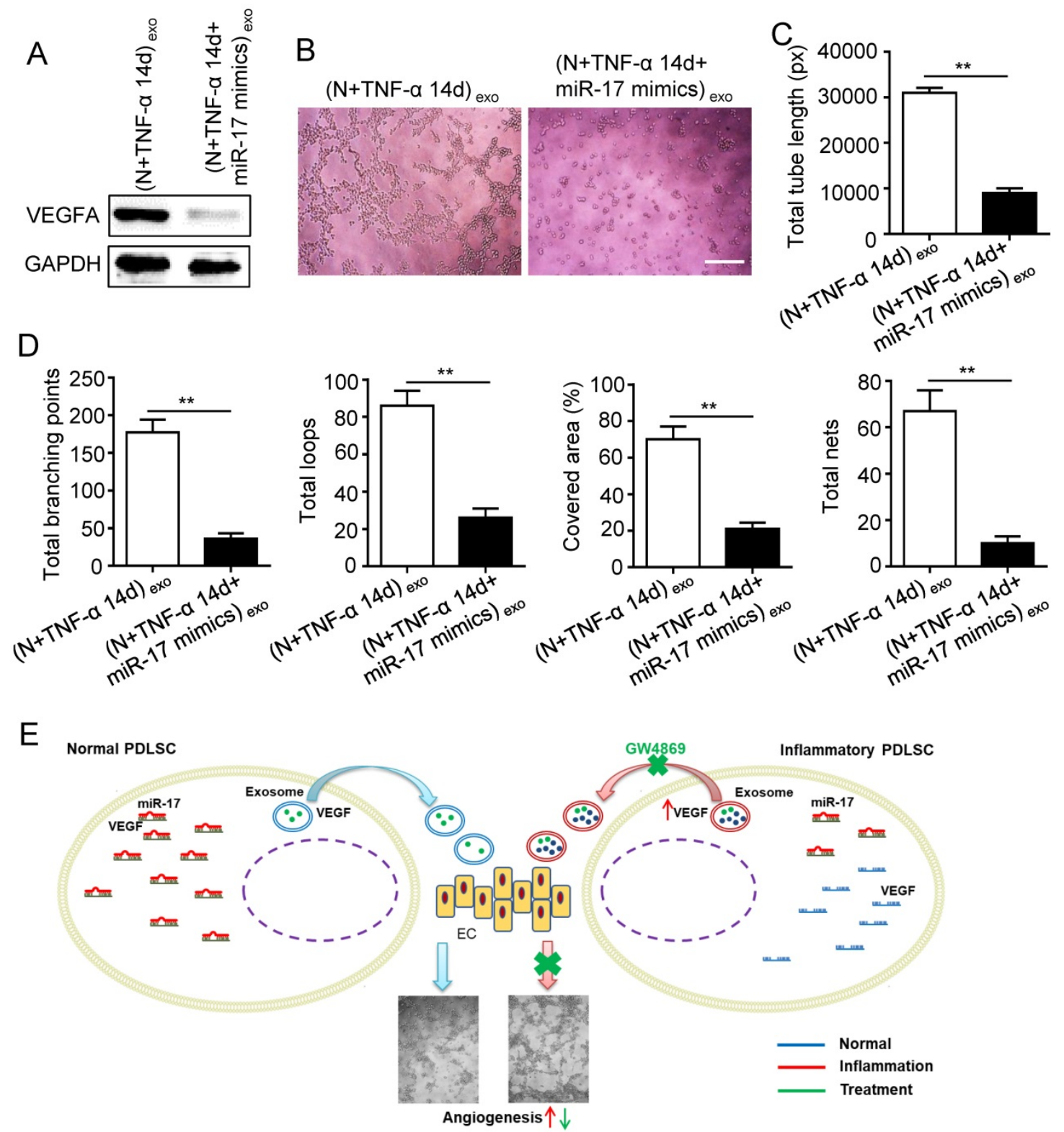

Figure 6. Effect of inflammation on angiogenesis via regulating exosome-transferred VEGF signaling of PDLSCs targeted by miR-17-5p. VEGFA expression (A) was detected in inflamed PDLSCs using western blot after transfecting miR-17 mimics, and tube formation (B) and total tube length, total branching points, total loops, covered area, total nets (C-D) were analyzed using Image J. (E) Schematic diagram of detailed mechanism. Scale bar $=200 \mu \mathrm{m}$. ** $<<0.01$. Unpaired two-tailed Student's t-test.

\section{Discussion}

Aberrant angiogenesis has been reported to be associated with the pathogenesis and progress of periodontitis [3, 5]. Functions of ECs compromised under inflammatory microenvironment of periodontitis [25]. Therefore, regulation of angiogenesis might be a candidate target for therapy of periodontitis. Gingiva and periodontal ligaments are neighbor tissues with close relationship, for example the main fibers of gingiva and periodontal ligaments cross over the alveolar ridge, the blood supply of periodontal ligaments partially derived from gingiva. Gingiva hyperemia is an obvious symptom of periodontitis, vascular alteration of gingiva has been investigated in the condition of periodontitis [5,6]. However, changes of vascularization of periodontal ligaments remain unknown. Therefore, it is significant to compare angiogenic conditions of these two tissues, and to determine the vascular alteration of periodontal ligaments to understand the mechanism of periodontitis and develop therapeutic approach. According to current study, periodontitis model was successfully established. Furthermore, similar to gingiva, vascularization of periodontal ligaments was also enhanced in periodontitis, suggesting that periodontal ligaments shared similar angiogenic disorder with gingiva under inflammation. However, the mechanism of vascular alteration remains unknown. 
PDLSCs are a type of dental stem cells residing in periodontal ligaments, which participate in regulation of periodontal tissues regeneration [26]. Apart from feature of osteogenic capacity, PDLSCs has been investigated to modulate angiogenesis of ECs [4, 9]. An indirect communication, such as paracrine, might be the crucial pathway involved in the mechanism, because PDLSCs and ECs could not directly interact with each other physiologically. Therefore, a transwell indirect co-culture system was employed to mimic cell-to-cell communication. Functions of PDLSCs are affected by inflammatory environment resulted from periodontitis, such as proliferative capacity, osteogenic potential and regulatory ability [27-29]. In our study, VEGFA, the vascular specific marker, was up-regulated in PDLSCs derived from periodontitis, suggesting that inflammation might affect the pro-angiogenesis of PDLSCs. Furthermore, we found that the pro-angiogenic ability of PDLSCs was improved under inflammatory condition with a time-dependent manner, which corresponded to the results of gradually enhanced vascularization in periodontitis [3, 5]. Thus, we confirmed that inflammatory micro-environment in periodontitis might be the key regulator on pro-angiogenesis of PDLSCs, and inflamed PDLSCs regulated HUVECs via paracrine dependent pathway.

Exosomes is a well-known paracrine mediator to execute mission of intercellular contact [13] and is associated with the change of micro-environment [30]. It has been reported that exosomes secretion and contents were under control of inflammatory microenvironment [31, 32]. We found that the secretion of exosomes was increased under inflammatory condition, indicating that the exosomes secretion was altered in PDLSCs of periodontitis. Furthermore, treatment with inflammatory exosomes promoted the angiogenesis of HUVECs, whereas blocking exosomes secretion abolished the pro-angiogenesis of inflamed PDLSCs. Therefore, we confirmed that inflammation promoted pro-angiogenesis of PDLSCs via exosomes mediated pathway.

Exosomes is a carrier to transport active factors intercellularly, such as functional protein and small RNA[13]. In current study, expression of VEGFA was increased in exosomes derived from inflamed PDLSCs, suggesting the important role of exosomeVEGFA in angionenic regulation. However, the up-stream signaling regulating VEGFA expression under inflammation remains elusive. MicroRNAs have been widely investigated to regulate vascularization through various signaling [33, 34]. MiR-17, a potential inhibitor of VEGF, has been reported to be associated with periodontal regeneration [21, 22, 24] and tissue angiogenesis [23].
Nevertheless, the role of miR-17 on pro-angiogenesis of PDSLCs in periodontitis remains unknown. We found that miR-17-5p was decreased under inflammation with a time-dependent manner, indicating a significant role of miR-17 in periodontitis. Additionally, the fact of miR-17-5p targeting on VEGFA was verified. Moreover, overexpression of miR-17-5p in inflamed PDLSCs resulted in decreased exosome-VEGFA and declined tube formation. Meanwhile, the effect of miR-17-5p on exosomes secretion was eliminated, confirming that the reduction of exosome-VEGFA credited to VEGFA suppression but not exosomes inhibition triggered by miR-17-5p. In addition, in our study, exosomes secretion and miR-17-5p/VEGFA signaling were both affected by inflammatory micro-environment, suggesting that up-regulation of exosomes secretion and VEGFA expression might synergistically contribute to increased vascularization of periodontal ligaments in periodontitis.

All the aforementioned data suggested that vascularization of periodontal ligaments was upregulated in periodontitis, which might credit to the fact that PDLSCs regulated angiogenesis of HUVECs via exosome-mediated transfer of VEGFA targeted by miR-17-5p.

\section{Abbreviations}

PDLSCs: periodontal ligament stem cells; VEGF: vascular endothelial growth factor; HUVECs: human umbilical vein endothelial cells; MSCs: Mesenchymal stem cells; miRNAs: micro-RNAs.

\section{Supplementary Material}

Supplementary figures.

http://www.medsci.org/v17p0558s1.pdf

\section{Acknowledgements}

This study was funded by the Key R \& D Plan Projects of Shaanxi Province (grant No.2017ZDXMSF-038); Natural Science Foundation of Jiangsu Province, China (grant No. BK20190250); Natural Science Foundation of China (grant No. 81970915, 31571532).

\section{Author Contributions}

Wenjia Liu, Zhang Zhang, Yi Shuai and Feng Zhou conceived and designed the study. Wenjia Liu and Zhang Zhang supervised the project. Zhang Zhang, Yi Shuai and Feng Zhou conducted the experiments, analyzed the data and wrote the manuscript. Jikai Yin and Jiachen $\mathrm{Hu}$ participated in sample collection and examination. Songlin Guo and Yan Wang were responsible for immunofluorescence staining. Wenjia Liu and Zhang Zhang reviewed and 
revised the manuscript.

\section{Competing Interests}

The authors have declared that no competing interest exists.

\section{References}

1. Kononen E, Gursoy M, Gursoy UK. Periodontitis: A Multifaceted Disease of Tooth-Supporting Tissues. Journal of clinical medicine. 2019; 8.

2. Rather HA, Jhala D, Vasita R. Dual functional approaches for osteogenesis coupled angiogenesis in bone tissue engineering. Materials science \& engineering C, Materials for biological applications. 2019; 103: 109761.

3. Gurav AN. The implication of periodontitis in vascular endothelial dysfunction. Eur J Clin Invest. 2014; 44: 1000-9.

4. Wei W, An Y, An Y, Fei D, Wang Q. Activation of autophagy in periodontal ligament mesenchymal stem cells promotes angiogenesis in periodontitis. J Periodontol. 2018; 89: 718-27.

5. Zoellner H, Hunter N. Vascular expansion in chronic periodontitis. J Oral Pathol Med. 1991; 20: 433-7.

6. Zoellner H, Chapple CC, Hunter N. Microvasculature in gingivitis and chronic periodontitis: disruption of vascular networks with protracted inflammation. Microsc Res Tech. 2002; 56: 15-31.

7. Mehrotra N, Singh S. Periodontitis. Treasure Island, US: StatPearls Publishing StatPearls Publishing LLC; 2019.

8. Lambrichts I, Driesen RB, Dillen Y, Gervois P, Ratajczak J, Vangansewinkel T, et al. Dental Pulp Stem Cells: Their Potential in Reinnervation and Angiogenesis by Using Scaffolds. J Endod. 2017; 43: S12-S6.

9. Yeasmin S, Ceccarelli J, Vigen M, Carrion B, Putnam AJ, Tarle SA, et al. Stem cells derived from tooth periodontal ligament enhance functional angiogenesis by endothelial cells. Tissue Eng Part A. 2014; 20: 1188-96.

10. Seo BM, Miura M, Gronthos S, Bartold PM, Batouli S, Brahim J, et al. Investigation of multipotent postnatal stem cells from human periodontal ligament. Lancet. 2004; 364: 149-55.

11. Gnecchi M, Danieli P, Malpasso G, Ciuffreda MC. Paracrine Mechanisms of Mesenchymal Stem Cells in Tissue Repair. Methods Mol Biol. 2016; 1416: 123-46.

12. Bruno S, Collino F, Tetta C, Camussi G. Dissecting paracrine effectors for mesenchymal stem cells. Adv Biochem Eng Biotechnol. 2013; 129: 137-52.

13. Hessvik NP, Llorente A. Current knowledge on exosome biogenesis and release. Cell Mol Life Sci. 2018; 75: 193-208.

14. Qin Y, Sun R, Wu C, Wang L, Zhang C. Exosome: A Novel Approach to Stimulate Bone Regeneration through Regulation of Osteogenesis and Angiogenesis. Int J Mol Sci. 2016; 17.

15. Zimta AA, Baru O, Badea M, Buduru SD, Berindan-Neagoe I. The Role of Angiogenesis and Pro-Angiogenic Exosomes in Regenerative Dentistry. Int J Mol Sci. 2019; 20.

16. Duran CL, Howell DW, Dave JM, Smith RL, Torrie ME, Essner JJ, et al. Molecular Regulation of Sprouting Angiogenesis. Comprehensive Physiology. 2017; 8: 153-235

17. Ng YS, Krilleke D, Shima DT. VEGF function in vascular pathogenesis. Exp Cell Res. 2006; 312: 527-37.

18. Schipani E, Maes C, Carmeliet G, Semenza GL. Regulation of osteogenesis-angiogenesis coupling by HIFs and VEGF. J Bone Miner Res. 2009; 24: 1347-53.

19. Lu L, Deegan A, Musa F, Xu T, Yang Y. The effects of biomimetically conjugated VEGF on osteogenesis and angiogenesis of MSCs (human and rat) and HUVECs co-culture models. Colloids Surf B Biointerfaces. 2018; 167: $550-9$

20. Sehic A, Tulek A, Khuu C, Nirvani M, Sand LP, Utheim TP. Regulatory roles of microRNAs in human dental tissues. Gene. 2017; 596: 9-18.

21. Li L, Liu W, Wang H, Yang Q, Zhang L, Jin F, et al. Mutual inhibition between HDAC9 and miR-17 regulates osteogenesis of human periodontal ligament stem cells in inflammatory conditions. Cell Death Dis. 2018; 9: 480

22. Liu $\mathrm{W}$, Liu $\mathrm{Y}$, Guo $\mathrm{T}$, Hu $\mathrm{C}$, Luo $\mathrm{H}$, Zhang $\mathrm{L}$, et al. TCF3, a novel positive regulator of osteogenesis, plays a crucial role in miR-17 modulating the diverse effect of canonical Wnt signaling in different microenvironments. Cell Death Dis. 2013; 4: e539.

23. Fiedler J, Thum T. New Insights Into miR-17-92 Cluster Regulation and Angiogenesis. Circ Res. 2016; 118: 9-11.

24. Liu Y, Liu W, Hu C, Xue Z, Wang G, Ding B, et al. MiR-17 modulates osteogenic differentiation through a coherent feed-forward loop in mesenchymal stem cells isolated from periodontal ligaments of patients with periodontitis. Stem Cells. 2011; 29: 1804-16

25. Lira-Junior R, Figueredo CM, Bouskela E, Fischer RG. Severe chronic periodontitis is associated with endothelial and microvascular dysfunctions: a pilot study. J Periodontol. 2014; 85: 1648-57.

26. Shuai Y, Ma Y, Guo T, Zhang L, Yang R, Qi M, et al. Dental Stem Cells and Tooth Regeneration. Adv Exp Med Biol. 2018; 1107: 41-52.

27. Zheng Y, Dong C, Yang J, Jin Y, Zheng W, Zhou Q, et al. Exosomal microRNA-155-5p from PDLSCs regulated Th17/Treg balance by targeting sirtuin-1 in chronic periodontitis. J Cell Physiol. 2019; 234: 20662-74.
28. Liu Y, Liu C, Zhang A, Yin S, Wang T, Wang Y, et al. Down-regulation of long non-coding RNA MEG3 suppresses osteogenic differentiation of periodontal ligament stem cells (PDLSCs) through miR-27a-3p/IGF1 axis in periodontitis. Aging (Albany NY). 2019; 11: 5334-50.

29. Wang $Y$, Sun $Y$, Zheng P, Cai C, Jiang $Y$, Zhang H, et al. Long non-coding RNAs mortal obligate RNA transcript regulates the proliferation of human periodontal ligament stem cells and affects the recurrence of periodontitis. Arch Oral Biol. 2019; 105: 1-4.

30. Hu C, Chen M, Jiang R, Guo Y, Wu M, Zhang X. Exosome-related tumor microenvironment. J Cancer. 2018; 9: 3084-92.

31. Zhao M, Dai W, Wang H, Xue C, Feng J, He Y, et al. Periodontal ligament fibroblasts regulate osteoblasts by exosome secretion induced by inflammatory stimuli. Arch Oral Biol. 2019; 105: 27-34.

32. Console L, Scalise M, Indiveri C. Exosomes in inflammation and role as biomarkers. Clin Chim Acta. 2019; 488: 165-71.

33. Tiwari A, Mukherjee B, Dixit M. MicroRNA Key to Angiogenesis Regulation: MiRNA Biology and Therapy. Curr Cancer Drug Targets. 2018; 18: 266-77.

34. Deng L, Bradshaw AC, Baker AH. Role of noncoding RNA in vascular remodelling. Current opinion in lipidology. 2016; 27: 439-48. 